\title{
Anisotropic States of Two-Dimensional Electron Systems in High Landau Levels: Effect of an In-Plane Magnetic Field
}

\author{
M. P. Lilly, ${ }^{1}$ K. B. Cooper,${ }^{1}$ J.P. Eisenstein, ${ }^{1}$ L. N. Pfeiffer, ${ }^{2}$ and K. W. West ${ }^{2}$ \\ ${ }^{1}$ California Institute of Technology, Pasadena, California 91125 \\ ${ }^{2}$ Bell Laboratories, Lucent Technologies, Murray Hill, New Jersey 07974
}

(Received 12 March 1999)

\begin{abstract}
We report the observation of an acute sensitivity of the anisotropic longitudinal resistivity of twodimensional electron systems in half-filled high Landau levels to the magnitude and orientation of an in-plane magnetic field. In the third and higher Landau levels, at filling fractions $\nu=9 / 2,11 / 2$, etc., the in-plane field can lead to a striking interchange of the "hard" and "easy" transport directions. In the second Landau level the normally isotropic resistivity and the weak $\nu=5 / 2$ quantized Hall state are destroyed by a large in-plane field and the transport becomes highly anisotropic.
\end{abstract}

PACS numbers: 73.20.Dx, 73.40.Kp, 73.50.Jt

In a recent paper, Lilly et al. [1] reported observations of several anomalies in the low temperature magnetotransport of high quality two-dimensional electron systems (2DES) when several Landau levels (LLs) are occupied. These anomalies include the development of large anisotropies and nonlinearities of the longitudinal resistivities ( $\rho_{x x}$ and $\rho_{y y}$ ) near half filling of several spin-resolved high LLs. These effects are observed only at very low temperatures $(T<150 \mathrm{mK})$ and when at least three or more LLs are occupied. Taken together, the observations offer strong evidence for new correlated many-electron states in high LLs which are different than the familiar fractional quantized Hall states found in the lowest $(N=0) \mathrm{LL}$. The fact that qualitatively identical phenomena are found in several adjacent LLs (having $N \geq 2$ ) points to a generic mechanism. In this paper we report on an investigation of the behavior of these unusual phenomena when a magnetic field component $B_{\|}$in the plane of the $2 \mathrm{D}$ system is added to the perpendicular field $B_{\perp}$ generating the Landau quantization. Our results show that the transport anisotropy can be quite sensitive to the in-plane magnetic field: in some cases $B_{\|}$appears to rotate the principal axes of the anisotropy by $90^{\circ}$.

Figure 1 illustrates the anisotropy of the longitudinal resistance seen in high LLs at $T=50 \mathrm{mK}$. The sample used to obtain these results is a modulation-doped GaAs/ AlGaAs heterojunction containing a 2DES with sheet density of $n_{s}=2.7 \times 10^{11} \mathrm{~cm}^{-2}$ and a mobility of $11 \times$ $10^{6} \mathrm{~cm}^{2} / \mathrm{V} \mathrm{s}$. This structure was grown by molecular beam epitaxy (MBE) on a $\langle 001\rangle$ GaAs substrate. The sample geometry (see insets) consists of a square mesa, $2.5 \mathrm{~mm}$ on a side, etched onto a larger square chip. The sides of the mesa are rotated $45^{\circ}$ relative to the natural cleavage directions $(\langle 110\rangle$ and $\langle 1 \overline{1} 0\rangle)$ of GaAs. Eight diffused indium Ohmic contacts are placed at the corners and midpoints of the sides of the square. For the data in Fig. 1a, the solid curve corresponds to current flowing between corner contacts along the diagonal of the square which is parallel to the $\langle 110\rangle$ crystallographic direction, while for the dotted curve the current flow is between corner contacts along the diagonal parallel to $\langle 1 \overline{1} 0\rangle$. The inset to the figures identifies these diagonals. For each trace, the measured voltage is that between the two midpoint contacts on one side of the current flow axis. These two orthogonal resistance measurements yield vastly different results when the 2DES Fermi level is near half filling of several spin resolved LLs. The largest anisotropy is observed in the $N=2$ LL near $\nu=9 / 2$ (where $\nu=n_{s} h / e B_{\perp}$ is the LL filling fraction). At this filling a deep minimum is seen when the current is driven along $\langle 110\rangle$, while a tall peak is found with the current along $\langle 1 \overline{1} 0\rangle$. For the data shown, the ratio of these resistances is about 60; in some samples we have found ratios as high as 3500 [2]. Substantial anisotropies are also seen at $\nu=11 / 2,13 / 2$, and several higher half-odd integers. Even after correcting for geometric effects [3] which exaggerate anisotropies observed in square samples, large intrinsic resistivity anisotropies (factor of $\sim 7$ for the $\nu=9 / 2$ data in Fig. 1) characterize transport in half filled high $(N \geq 2)$ LLs. This contrasts sharply with the essentially isotropic transport observed at $\nu=7 / 2$ (shown in Fig. 1) and 5/2 in the $N=1 \mathrm{LL}$ and at $\nu=3 / 2$ in the $N=0$ lowest LL. Figure 1 b demonstrates that for currents applied between opposing midpoints of the square, i.e., along the $\langle 100\rangle$ and $\langle 010\rangle$ directions, little

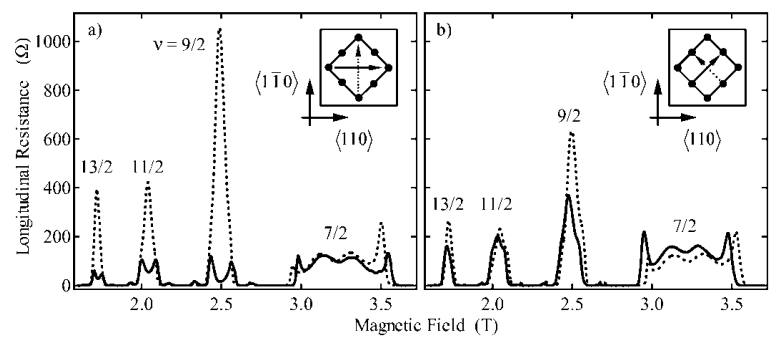

FIG. 1. Transport anisotropy at $T=50 \mathrm{mK}$ in high Landau levels in a diamond-shaped mesa. (a) current flow along $\langle 1 \overline{1} 0\rangle$ (dotted) and $\langle 110\rangle$ (solid). (b) current flow along $\langle 010\rangle$ (dotted) and $\langle 100\rangle$ (solid). 
anisotropy is seen at any filling factor. Collectively, these results show that the principal axes of the transport anisotropy are roughly aligned along the $\langle 110\rangle$ and $\langle 1 \overline{1} 0\rangle$ directions. We emphasize that the anisotropy axes observed in this "diamond" mesa are precisely the same as was found earlier [1] using a simple cleaved square (from the same parent MBE wafer) having edges along $\langle 110\rangle$ and $\langle 1 \overline{1} 0\rangle$. This demonstrates that the orientation of the boundaries of the 2DES does not influence the orientation of the anisotropy.

Let us now turn to the effect of an added in-plane magnetic field $B_{\|}$. Given the large anisotropies observed near $\nu=9 / 2,11 / 2,13 / 2$, etc., it is essential to examine not only the dependence on the magnitude of $B_{\|}$, but also on its direction. Consequently, we here report the results of separate experiments with $B_{\|}$oriented along $\langle 110\rangle$ and $\langle 1 \overline{1} 0\rangle$. Four different cooldowns from room temperature were required, two for each field orientation. In each case, the magnitude of $B_{\|}$was adjusted in situ at low temperature by tilting the sample relative to an applied magnetic field. Obviously, the perpendicular component of the field $B_{\perp}$ is constant during studies of a given Landau level filling fraction.

Figure 2 shows the effect of tilting the sample on the longitudinal resistance in the vicinity of $\nu=9 / 2$ when the in-plane magnetic field lies along $\langle 110\rangle$. For these and all subsequent data in this paper, the sample geometry is a simple $5 \times 5 \mathrm{~mm}^{2}$ cleaved square with contacts on the corners and at the midpoints of the sides. $R_{x x}$ refers to the resistance observed with the current flowing between midpoint contacts on opposite sides of the square along a line parallel to the $\langle 1 \overline{1} 0\rangle$ direction. The measured voltage difference is that between corner contacts on one side of the current flow axis. Similarly, $R_{y y}$ refers to the same basic configuration but rotated by $90^{\circ}$ so that the average current flow is along the $\langle 110\rangle$ direction. The three panels in the figure show these resistances at $T=50 \mathrm{mK}$ plotted versus perpendicular magnetic field $B_{\perp}$ at tilt angles $\theta=0$, $11^{\circ}$, and $34^{\circ}$. The figure reveals that the large peak in $R_{x x}$ at $\nu=9 / 2$ seen at $\theta=0$ is rapidly suppressed as the

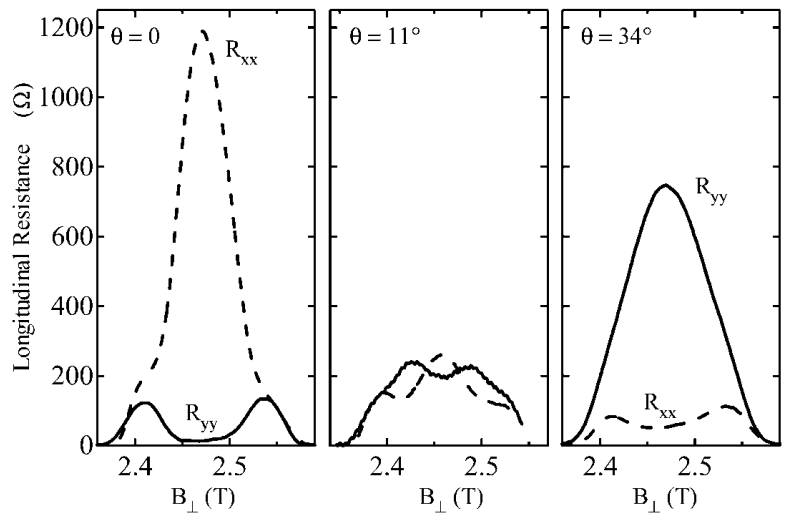

FIG. 2. Interchange of resistance anisotropy at $\nu=9 / 2$ at $T=50 \mathrm{mK}$ due to an in-plane magnetic field along $\langle 110\rangle$. sample is tilted. Eventually, the peak in $R_{x x}$ is replaced by a minimum. At the same time, the deep minimum in $R_{y y}$ at $\theta=0$ behaves in essentially the opposite fashion: it transforms into a tall peak. At around $\theta=11^{\circ}$ the two resistance traces are comparable in magnitude throughout the range $4<\nu<5$. By $\theta=34^{\circ}$, however, the resistance at $\nu=9 / 2$ is again strongly anisotropic, but with principal axes that are rotated by $90^{\circ}$ in the $2 \mathrm{D}$ plane relative to their orientation at $\theta=0$. Thus, the addition of a sufficiently large in-plane magnetic field directed along $\langle 110\rangle$ changes the "hard" transport direction (i.e., high resistance) at $\nu=9 / 2$ from $\langle 1 \overline{1} 0\rangle$ to $\langle 110\rangle$ and the "easy" direction (low resistance) from $\langle 110\rangle$ to $\langle 1 \overline{1} 0\rangle$.

We find that tilting produces a similar "interchange effect" on the anisotropic resistances at all half-odd integer filling from $\nu=9 / 2$ to $21 / 2$ (and possibly beyond) provided that $B_{\|}$is directed along $\langle 110\rangle$. This is demonstrated by the left-hand panels of Fig. 3 where values of $R_{x x}$ and $R_{y y}$ at $\nu=9 / 2,11 / 2,13 / 2$, and $15 / 2$ are plotted versus $B_{\|}$. The figure shows that in each case the $R_{x x}>R_{y y}$ anisotropy seen at $B_{\|}=0$ (i.e., at $\theta=0$ ) gives way to the opposite condition, $R_{x x}<R_{y y}$, at large $B_{\|}$. Interestingly, the data also show that the crossover point (where $R_{x x} \approx R_{y y}$ ) occurs at approximately the same inplane field, $B_{\|} \approx 0.5 \mathrm{~T}$, at each filling factor.

The right-hand panels of Fig. 3 show that tilting with $B_{\|}$ directed along the $\langle 1 \overline{1} 0\rangle$ direction produces very different results than with $B_{\|}$along $\langle 110\rangle$. At $\nu=9 / 2$, the resistances $R_{x x}$ and $R_{y y}$ approach one another and even cross but, unlike the case when $B_{\|}$is along $\langle 110\rangle$, no significant
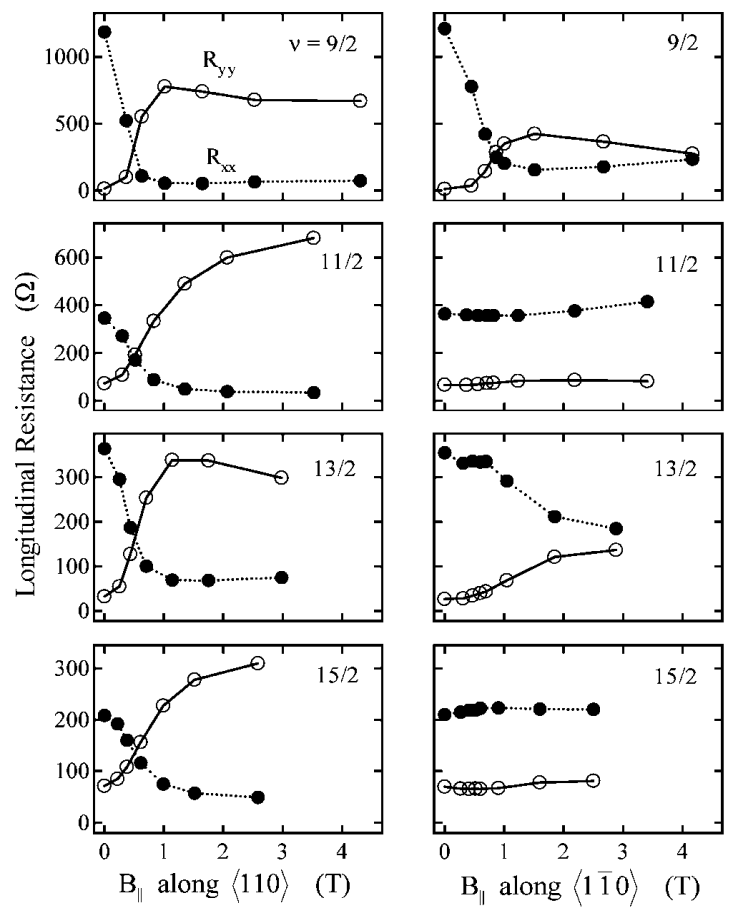

FIG. 3. Dependence of $R_{x x}$ and $R_{y y}$ at $\nu=9 / 2-15 / 2$ on an in-plane magnetic field at $T=50 \mathrm{mK}$. Left panels: $B_{\|}$along $\langle 110\rangle$. Right panels: $B_{\|}$along $\langle 1 \overline{1} 0\rangle$. 
anisotropy develops at large tilt angle. At the higher halfodd integer fillings the difference between the two different orientations of $B_{\|}$is even more striking. At $\nu=11 / 2$, for example, applying $B_{\|}$along $\langle 1 \overline{1} 0\rangle$ has hardly any effect at all, while for $B_{\|}$along $\langle 110\rangle$ the resistances interchange dramatically and lead to an anisotropy ratio at large tilt angles that exceeds its value at $\theta=0$. This very different behavior between $\nu=9 / 2$ and $11 / 2$ is particularly interesting since these two filling factors belong to the same $N=2$ orbital LL. In the $N=3 \mathrm{LL}$ applying $B_{\|}$along $\langle 1 \overline{1} 0\rangle$ does have a substantial effect on $\nu=13 / 2$ but it is not nearly so strong as the interchange observed when $B_{\|}$is along $\langle 110\rangle$. At $\nu=15 / 2$, like $\nu=11 / 2$, there is little or no effect with $B_{\|}$along $\langle 1 \overline{1} 0\rangle$. Higher half-odd integers (e.g., $\nu=17 / 2,19 / 2$, and 21/2) also show virtually no dependence upon an in-plane magnetic field directed along $\langle 1 \overline{1} 0\rangle$.

In some cases, e.g., $\nu=11 / 2,15 / 2,19 / 2$, etc., the inplane magnetic field appears to exert a "torque" on the anisotropy axes. No effect is seen for $B_{\|}$along $\langle 1 \overline{1} 0\rangle$ but a large effect, which ultimately interchanges the "easy" and "hard" transport directions, is observed when $B_{\|}$is along $\langle 110\rangle$. This interchange results in the high resistance or "hard" transport direction being parallel to $B_{\|}$. It is tempting to suggest that for these filling factors the anisotropy axes smoothly rotate as $B_{\|}$is applied along $\langle 110\rangle$. We would then expect to find some intermediate value of $B_{\|}$where these axes are rotated by $45^{\circ}$ relative to their $B_{\|}=0$ orientation. We searched for this by performing transport measurements in which the current was driven along $\langle 100\rangle$ and $\langle 010\rangle$ using corner contacts along diagonals to the square. At no intermediate value of $B_{\|}$was a large anisotropy detected along these directions.

As Lilly et al. [1], and subsequently, Du et al. [4] observed, the anisotropic transport in half-filled high LLs is a very low temperature $(T<150 \mathrm{mK})$ phenomenon. This largely remains the case in the presence of the inplane magnetic field. At very large $B_{\|}$there is some evidence that the interchanged anisotropy can persist to higher temperatures than at $\theta=0$ but this is an issue best left to a future publication.

Transport near half filling of high LLs is, in addition to anisotropic, substantially nonlinear. Lilly et al. [1] observed that the large resistivity peak in $R_{x x}$ grows substantially if a dc current is added to the small ac excitation. Similarly, $R_{y y}$ falls when the dc current is applied. While the $R_{x x}$ nonlinearity is clearly inconsistent with electron heating, the situation is not as clear for $R_{y y}$. In any case, we report here that these nonlinearities also interchange in the same way as the resistances themselves do when an in-plane field is applied.

The origin of the effects of an in-plane magnetic field on the anisotropic states of high mobility 2D electron systems at half filling of highly excited LLs is not understood. This is not surprising since the origin of the large anisotropy in high LLs, even without any in-plane magnetic field, is itself not understood. Interesting suggestions, based upon
Hartree-Fock calculations, of unidirectional charge density waves ("stripes") have been advanced [5,6], but quantum fluctuations are predicted to be severe and to possibly break up the stripes and produce liquid crystalline behavior [7]. Recent numerical calculations [8] have shown that near half filling of high LLs 2D electron systems become extremely susceptible to periodic potential modulations having wavelengths comparable to the cyclotron radius. What is clear from the experiments is that under the appropriate conditions (i.e., very high sample mobility, very low temperatures, and half filling of the third and higher LLs) 2D electron systems develop a macroscopic transport anisotropy. The evidence strongly suggests that this is an intrinsically many-electron effect. It is also clear that if the many-electron state has lost full rotational symmetry, some mechanism must exist for orienting that state over the macroscopic $(\sim \mathrm{mm})$ dimensions of our samples. All of the GaAs heterostructure samples we have studied (11 from 7 different MBE wafers) show, in the absence of tilting, the same anisotropy axes. The orientation of these axes appears to be immune to thermal cycling to room temperature, magnetic field reversal, and changing the geometry of the 2DES (e.g., the diamond mesa discussed above). These facts suggest that the anisotropy axes may be determined by something internal to the GaAs heterostructure, at least in the absence of an inplane magnetic field. Possibilities include known growth instabilities which lead to oblong islands on the sample surface oriented along the $\langle 1 \overline{1} 0\rangle$ crystal direction [9] and anisotropies in the GaAs band structure owing to the lack of inversion symmetry at the heterointerface [10].

An in-plane magnetic field $B_{\|}$couples to a $2 \mathrm{D}$ electron gas through the electron spin and via the finite thickness of the electron wave function in the direction normal to the $2 \mathrm{D}$ plane. In the former case, $B_{\|}$adds to the already significant spin Zeeman energy produced by the perpendicular magnetic field $B_{\perp}$. The high sensitivity of the transport coefficients to relatively small $B_{\|}$(e.g., at $\nu=$ $9 / 2$ the 60 -fold resistance anisotropy at $\theta=0$ is wiped out entirely by $\theta \approx 11^{\circ}$ where $B_{\|} / B_{\perp} \approx 0.19$ ) suggests that small increases in the spin flip energy are not the most important effect of the in-plane field. On the other hand, the in-plane field itself leads to mixing of the different subbands of the heterostructure confinement potential. In the presence of the perpendicular field, this subband mixing is accompanied by LL mixing. The cyclotron orbits become anisotropically distorted [11] by the in-plane field. Since the resistivity of the 2D system is highly anisotropic at $B_{\|}=0$, it is plausible that such distortions could lead to a sensitivity to the direction, as well as the magnitude, of the in-plane magnetic field.

Since the in-plane magnetic field breaks the rotational invariance of the system, it is important to examine the dependence of the longitudinal resistance on the magnitude and direction of the in-plane magnetic field in situations other than high half-odd integer filling factors. For example, in the Shubnikov-de Haas regime at low 
perpendicular magnetic fields we find that the addition of an in-plane field has little effect. We find that at $B_{\perp} \approx 0.5 \mathrm{~T}$ the application of $B_{\|} \approx 0.87 \mathrm{~T}$ along either the $\langle 1 \overline{1} 0\rangle$ or $\langle 110\rangle$ axes changes the resistances, and their ratio $R_{x x} / R_{y y}$, by only about $10 \%$. This contrasts sharply with the situation at $\nu=9 / 2,11 / 2$, etc., where, as Fig. 3a shows, the application of this much in-plane field can change this ratio by almost 3 orders of magnitude. Similarly, at high magnetic field, at $\nu=3 / 2$ in the $N=0$ lowest LL, we also find relatively little effect of an in-plane field. Applying $B_{\|} \approx 7.7 \mathrm{~T}$ along either $\langle 110\rangle$ or $\langle 1 \overline{1} 0\rangle$ yields only a weak increase $(\sim 30 \%)$ of the resistances $R_{x x}$ and $R_{y y}$ at $T=50 \mathrm{mK}$. In-plane fields of this magnitude have an enormous impact at $\nu=9 / 2$.

The remaining case to discuss is the $N=1$ first excited LL. This is the location of the still enigmatic $\nu=$ $5 / 2$ fractional quantized Hall effect (FQHE). As we have stressed, in the absence of tilting, no dramatic transport anisotropies appear near half filling of either spin branch of this LL (i.e., at $\nu=5 / 2$ or $7 / 2$ ). Indeed, the contrast between the giant low temperature anisotropy of $\nu=9 / 2$ in the $N=2 \mathrm{LL}$ and these essentially isotropic neighbors in the $N=1$ level is one of the most striking results reported by Lilly et al. Previous experiments [12] have shown that tilting steadily weakens the $\nu=5 / 2$ FQHE state. We have corroborated this result using our present sample by examining the temperature development of $R_{x x}$ and $R_{y y}$ at $\nu=5 / 2$. On the other hand, as Fig. 4 shows, the resistivity in the vicinity of $\nu=5 / 2$ and $7 / 2$ becomes strongly anisotropic as the sample is tilted. The orientation of this anisotropy is determined largely by the direction of the in-plane field: the resistance measured with current flow parallel to $B_{\|}$increases steadily upon tilting, while for currents flowing perpendicular to $B_{\|}$the resistance changes only slightly. The resulting anisotropies can be quite significant: at $\nu=5 / 2$ and $B_{\|}=7.7 \mathrm{~T}$ along $\langle 110\rangle$ we find $R_{y y} \approx 35 R_{x x}$. Since the magnitude of this $B_{\|}$-induced anisotropy at $\nu=5 / 2$ and $7 / 2$ is generally stronger for $B_{\|}$along $\langle 110\rangle$ than $\langle 1 \overline{1} 0\rangle$ there may also be some sensitivity to the internal crystal axes. These new results suggest that the correlation physics operative at $\nu=9 / 2$ might be becoming relevant at $\nu=5 / 2$ and $7 / 2$. In some sense, the $N=1 \mathrm{LL}$ is straddling the fence between the FQHE regime in the lowest LL and the spontaneously anisotropic non-FQHE states which dominate in the $N \geq 2 \mathrm{LL}$. If the fragile $\nu=5 / 2$ state is destroyed by tilting, whether through a spin Zeeman or some other effect, the real question is what takes its place at high $B_{\|}$? Evidently the answer is something anisotropic; perhaps more akin to the $\nu=9 / 2$ state in the $N=2$ LL than the composite fermion liquid which is believed to describe the $\nu=3 / 2$ state in the $N=0$ level [13,14].

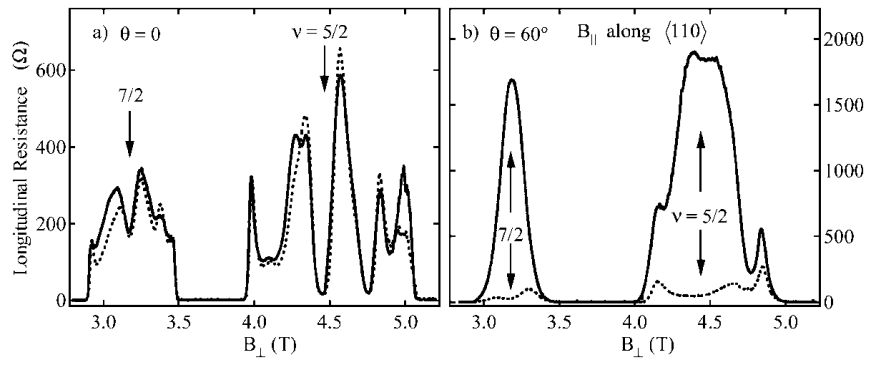

FIG. 4. Transport at $20 \mathrm{mK}$ in the $N=1 \mathrm{LL}$. Tilting destroys the $\nu=5 / 2$ and $7 / 2$ FQHE states and leaves the transport highly anisotropic. Current along $\langle 110\rangle$ (solid) and $\langle 1 \overline{1} 0\rangle$ (dotted); $B_{\|}$along $\langle 110\rangle$. Note the different vertical scales.

In summary, we have reported on the effect of an inplane magnetic field on transport in high Landau levels. In the third and higher Landau levels, at filling fractions $\nu=9 / 2,11 / 2,13 / 2$, etc., the recently discovered strongly anisotropic many-electron states near half filling are found to be very sensitive to the in-plane field. Depending upon the direction of the in-plane field, the hard and easy transport directions can actually interchange. In the $N=1$ Landau level, we find that the in-plane field not only destroys the weak $\nu=5 / 2$ and $7 / 2$ fractional quantized Hall states, but also replaces them with a strongly anisotropic resistivity.

We are indebted to S. Girvin, A.H. MacDonald, B. Shklovskii, S. Simon, and K. Yang for valuable discussion and the NSF for Grant No. DMR-9700945.

[1] M. P. Lilly et al., Phys. Rev. Lett. 82, 394 (1999).

[2] M.P. Lilly et al., cond-mat/9903153.

[3] S. H. Simon, cond-mat/9903086.

[4] R. R. Du et al., Solid State Commun. 109, 389 (1999).

[5] A. A. Koulakov, M. M. Fogler, and B. I. Shklovskii, Phys. Rev. Lett. 76, 499 (1996); Phys. Rev. B 54, 1853 (1996).

[6] R. Moessner and J. Chalker, Phys. Rev. B 54, 5006 (1996).

[7] E. Fradkin and S. Kivelson, Phys. Rev. B 59, 8065 (1999).

[8] E. H. Rezayi, F. D. M. Haldane, and K. Yang, cond-mat/ 9903258.

[9] C. Orme et al., Appl. Phys. Lett. 64, 860 (1994).

[10] H. Kroemer, cond-mat/9901016.

[11] T. Jungwirth, A.H. MacDonald, L. Smrcka, and S. M. Girvin, cond-mat/9905353; B. Shklovskii and M. Fogler (private communications); T. Stanescu, I. Martin, and P. Phillips, cond-mat/9905116.

[12] J. P. Eisenstein et al., Phys. Rev. Lett. 61, 997 (1988).

[13] For reviews of the fractional quantum Hall and composite fermion problems, see Perspectives in Quantum Hall Effects, edited by S. Das Sarma and A. Pinczuk (John Wiley, New York, 1997).

[14] R. H. Morf, Phys. Rev. Lett. 80, 1505 (1998). 